\title{
On Combining Neighbouring Extremals with Control Parameterization
}

\author{
Chongyang LIU ${ }^{1}$, Qun LIN $^{2}$, Ryan LOXTON ${ }^{2,3}$, Kok Lay TEO ${ }^{2}$ \\ 1. School of Mathematics and Information Science, Shandong Institute of Business and Technology, Yantai 264005 P. R. China \\ E-mail: liu_chongyang@yahoo.com \\ 2. Department of Mathematics and Statistics, Curtin University, Perth 6102 Australia \\ E-mail: q.lin@curtin.edu.au;r.loxton@curtin.edu.au;k.1.teo@curtin.edu.au \\ 3. Institute of Cyber-Systems and Control, Zhejiang University, Hangzhou 310027 P. R. China \\ E-mail: r.loxton@curtin.edu.au
}

\begin{abstract}
In this paper, we consider the neighbouring extremals for a class of optimal control problems with control constraints. We first solve the optimal control problem using control parameterization method to obtain the optimal open-loop control and the optimal reference state. Then, a neighbouring feedback control law is derived for small state perturbations caused by changes on reference state at switching times.
\end{abstract}

Key Words: Nonlinear system, Optimal feedback control, Control parameterization, Neighbouring extremals

\section{Introduction}

In general, optimal control problems, which are considerably complex and involve a dynamic system. There are very few real-world optimal control problems that lend themselves to analytical solutions. As a result, using numerical algorithms to solve the optimal control problems becomes a common approach [1-4]. Among these is the control parameterization method $[4,5]$, which discretizes the control space by approximating the control by a piecewise-constant function and thereby yields an approximate nonlinear programming problem. This approximate problem can then be solved efficiently to obtain open-loop optimal controls using standard gradient-based optimization techniques [6]. However, it is well known that the open-loop optimal control is very sensitive to disturbances due to uncertainty in the initial conditions or model equations. Thus, a feedback controller is much superior in practice. The task of fully synthesizing a closed-loop optimal control is, however, very difficult since the solution of a nonlinear Hamilton-Jacobi-Bellman partial differential equation is required [7].

The neighboring extremal method provides a first-order approximation to the optimal solution corresponding to the disturbance [8]. For unconstrained optimal control problem$\mathrm{s}$, a neighbouring extremal solution was derived using second variation for situation where only the initial and/or terminal conditions are perturbed [9]. This method is extended to dynamic optimization problems with perturbations in additional parameter vector [10]. For constrained optimal control problems, a method of computing neighbouring extremal for nonlinear systems with control constraints is developed [11]. Furthermore, neighbouring extremal solution$\mathrm{s}$ are obtained for nonlinear systems with state constraints [12-16]. However, the above mentioned methods are based on the assumption that a continuous optimal control can be obtained exactly.

In this paper, we consider a class of nonlinear optimal

This work is supported by National Natural Science Foundation of China (NSFC) under grants 11201267 and 11350110208, and the Australian Research Council (ARC) under grant DP110100083. control problems subject to control constraints. We assume that an initial condition for the dynamic systems is specified. Then, we obtain a reference open-loop optimal piecewise constant control using the control parameterization method [5]. Furthermore, a neighbouring control law in a state feedback form is derived for small state perturbations caused by changes on reference state at switching times.

\section{Optimal Control Problem}

Consider the following nonlinear control system:

$$
\begin{aligned}
\dot{\boldsymbol{x}}(t) & =\boldsymbol{f}(t, \boldsymbol{x}(t), \boldsymbol{u}(t)), \quad t \in[0, T], \\
\boldsymbol{x}(0) & =\boldsymbol{x}^{0}
\end{aligned}
$$

where $\boldsymbol{x}(t) \in \mathbb{R}^{n}$ is the system's state at time $t, \boldsymbol{u}(t) \in \mathbb{R}^{m}$ is the system's control signal at time $t, \boldsymbol{x}^{0}$ is a given initial state, $T>0$ is a given terminal time, and $f: \mathbb{R} \times \mathbb{R}^{n} \times$ $\mathbb{R}^{m} \rightarrow \mathbb{R}^{n}$ is a given continuously differentiable function.

We suppose that the control signal is subject to the following constraints:

$$
\phi(\boldsymbol{u}(t)) \leq \mathbf{0}, \quad t \in[0, T],
$$

where $\phi: \mathbb{R}^{m} \rightarrow \mathbb{R}^{q}$ is a given continuously differentiable function. Any measurable function $\boldsymbol{u}:[0, T] \rightarrow \mathbb{R}^{m}$ such that (3) holds for almost all $t \in[0, T]$ is called a feasible control. Let $\mathcal{F}$ be the class of all such feasible controls.

Assume that system (1)-(2) has a unique solution $\boldsymbol{x}(\cdot \mid \boldsymbol{u})$ corresponding to each $\boldsymbol{u} \in \mathcal{F}$ on $[0, T]$. We now state our optimal control problem as follows.

Problem P. Choose a feasible control $\boldsymbol{u} \in \mathcal{F}$ to minimize the cost functional

$$
J(\boldsymbol{u})=\Phi(\boldsymbol{x}(T \mid \boldsymbol{u}))+\int_{0}^{T} f_{0}(t, \boldsymbol{x}(t \mid \boldsymbol{u}), \boldsymbol{u}(t)) d t,
$$

where $\Phi: \mathbb{R}^{n} \rightarrow \mathbb{R}$ and $f_{0}: \mathbb{R} \times \mathbb{R}^{n} \times \mathbb{R}^{m} \rightarrow \mathbb{R}$ are given continuously differentiable functions.

Note that Problem P is a general form of optimal control problems subject to control constraints. 


\section{Control Parameterization}

Generally speaking, Problem $\mathrm{P}$ is too complicated to solve analytically. Thus, in practice, an approximation of Problem $\mathrm{P}$ is obtained using the control parameterization method [5].

Let $p \geq 2$ be a given integer. Furthermore, let $\tau_{i}, i=$ $0,1, \ldots, p$, be given partition points of $[0, T]$ such that

$$
0=\tau_{0}<\tau_{1}<\tau_{2}<\cdots<\tau_{p-1}<\tau_{p}=T .
$$

For given vectors $\boldsymbol{\sigma}^{i} \in \mathbb{R}^{m}, i=1, \ldots, p$, define

$$
\left(\boldsymbol{\sigma}^{1}, \ldots, \boldsymbol{\sigma}^{p}\right)=\left[\left(\boldsymbol{\sigma}^{1}\right)^{\top}, \ldots,\left(\boldsymbol{\sigma}^{p}\right)^{\top}\right]^{\top} \in \mathbb{R}^{p m} .
$$

We approximate the control $\boldsymbol{u}$ by the following piecewiseconstant function:

$$
\boldsymbol{u}(t) \approx \boldsymbol{u}^{p}(t)=\sum_{i=1}^{p} \boldsymbol{\sigma}^{i} \chi_{\left[\tau_{i-1}, \tau_{i}\right)}(t), \quad t \in[0, T),
$$

where $\chi_{\left[\tau_{i-1}, \tau_{i}\right)}: \mathbb{R} \rightarrow \mathbb{R}$ is the characteristic function defined by

$$
\chi_{\left[\tau_{i-1}, \tau_{i}\right)}(t)= \begin{cases}1, & \text { if } t \in\left[\tau_{i-1}, \tau_{i}\right), \\ 0, & \text { otherwise. }\end{cases}
$$

Substituting the piecewise-constant control (7) into the dynamic system (1)-(2) yields

$$
\begin{aligned}
\dot{\boldsymbol{x}}(t) & =\sum_{i=1}^{p} \boldsymbol{f}\left(t, \boldsymbol{x}(t), \boldsymbol{\sigma}^{i}\right) \chi_{\left[\tau_{i-1}, \tau_{i}\right)}(t), \quad t \in[0, T], \\
\boldsymbol{x}(0) & =\boldsymbol{x}^{0}
\end{aligned}
$$

Under the control approximation (7), the control constrain$\mathrm{t}$ (3) becomes

$$
\phi\left(\boldsymbol{\sigma}^{i}\right) \leq \mathbf{0}, \quad i=1, \ldots, p .
$$

Let $\Xi$ denote the set of all control value vectors $\boldsymbol{\sigma}=$ $\left(\boldsymbol{\sigma}^{1}, \ldots, \boldsymbol{\sigma}^{p}\right)$ satisfying the constraints (11). Furthermore, let $\mathcal{A}(i \mid \boldsymbol{\sigma}) \subseteq\{1, \ldots, q\}$ be the index set defining the active constraints at the point $\sigma^{i}$, that is,

$$
\mathcal{A}(i \mid \boldsymbol{\sigma})=\left\{j \mid \phi_{j}\left(\boldsymbol{\sigma}^{i}\right)=0\right\} .
$$

Assume that $\boldsymbol{x}^{p}(\cdot \mid \boldsymbol{\sigma})$ is the solution of system (9)-(10) corresponding to each $\sigma \in \Xi$ on $[0, T]$. We may now specify the approximate problem as follows.

$\operatorname{Problem} \mathbf{P}(p)$. Choose a control value vector $\boldsymbol{\sigma} \in \Xi$ to minimize the cost functional

$$
J^{p}(\boldsymbol{\sigma})=\Phi\left(\boldsymbol{x}^{p}(T \mid \boldsymbol{\sigma})\right)+\sum_{i=1}^{p} \int_{\tau_{i-1}}^{\tau_{i}} f_{0}\left(t, \boldsymbol{x}^{p}(t \mid \boldsymbol{\sigma}), \boldsymbol{\sigma}^{i}\right) d t .
$$

\section{The Optimal Reference and Neighbouring Tra- jectories}

\subsection{Optimal Reference Trajectory}

Problem $\mathrm{P}(p)$ can be numerically solved by gradient-based optimization methods [4]. Let $\sigma^{*}$ be the optimal control value vector and $\boldsymbol{x}^{p *}(\cdot)=\boldsymbol{x}^{p}\left(\cdot \mid \boldsymbol{\sigma}^{*}\right)$ be the optimal reference trajectory of Problem $\mathrm{P}(p)$, respectively. Furthermore, let $\mathcal{H}$ and $\mathcal{L}$ be Hamiltonian and Lagrangian functions defined, respectively, by

$$
\begin{aligned}
\mathcal{H}\left(t, \boldsymbol{x}^{p}, \boldsymbol{\sigma}, \boldsymbol{\lambda}\right)= & \sum_{i=1}^{p} H\left(t, \boldsymbol{x}^{p}, \boldsymbol{\sigma}^{i}, \boldsymbol{\lambda}\right) \chi_{\left[\tau_{i-1}, \tau_{i}\right)}(t), \\
\mathcal{L}\left(\boldsymbol{x}^{p}, \boldsymbol{\sigma}, \boldsymbol{\lambda}, \boldsymbol{\rho}\right)= & \sum_{i=1}^{p}\left\{\int_{\tau_{i-1}}^{\tau_{i}} H\left(t, \boldsymbol{x}^{p}, \boldsymbol{\sigma}^{i}, \boldsymbol{\lambda}\right) d t\right. \\
& \left.+\left(\boldsymbol{\rho}^{i}\right)^{\top} \boldsymbol{\phi}\left(\boldsymbol{\sigma}^{i}\right)\right\}
\end{aligned}
$$

where $\boldsymbol{\rho}=\left[\left(\boldsymbol{\rho}^{1}\right)^{\top}, \ldots,\left(\boldsymbol{\rho}^{p}\right)^{\top}\right]^{\top}, \boldsymbol{\rho}^{i}=\left[\rho_{1}^{i}, \ldots, \rho_{q}^{i}\right]^{\top}$ and

$H(t, \boldsymbol{x}, \boldsymbol{u}, \boldsymbol{\lambda})=f_{0}(t, \boldsymbol{x}(t), \boldsymbol{u}(t))+(\boldsymbol{\lambda}(t))^{\top} \boldsymbol{f}(t, \boldsymbol{x}(t), \boldsymbol{u}(t))$.

It follows from gradient formulae [5] and Karush-KuhnTucker optimality conditions [6] that there exists a continuous vector $\boldsymbol{\lambda}^{*}(t) \in \mathbb{R}^{n}$ and multiplier $\boldsymbol{\rho}^{*} \in \mathbb{R}^{p q}$ such that $\boldsymbol{\sigma}^{*}, \boldsymbol{x}^{p *}(\cdot), \boldsymbol{\lambda}^{*}(\cdot)$ and $\boldsymbol{\rho}^{*}$ meet all the following necessary conditions for optimality:

$$
\begin{aligned}
& \dot{\boldsymbol{x}}^{p *}(t)=\sum_{i=1}^{p} \boldsymbol{f}\left(t, \boldsymbol{x}^{p *}(t), \boldsymbol{\sigma}^{* i}\right) \chi_{\left[\tau_{i-1}, \tau_{i}\right)}(t) \\
& \boldsymbol{x}^{p *}(0)=\boldsymbol{x}^{0} \\
& \left(\dot{\boldsymbol{\lambda}}^{*}(t)\right)^{\top}=-\frac{\partial \mathcal{H}\left(t, \boldsymbol{x}^{p *}, \boldsymbol{\sigma}^{*}, \boldsymbol{\lambda}^{*}\right)}{\partial \boldsymbol{x}} \\
& \left(\boldsymbol{\lambda}^{*}(T)\right)^{\top}=\frac{\partial \Phi\left(\boldsymbol{x}^{p *}(T)\right)}{\partial \boldsymbol{x}}, \\
& \boldsymbol{\lambda}^{*}\left(\tau_{\iota}-\right)=\boldsymbol{\lambda}^{*}\left(\tau_{\iota}+\right), \quad \iota=1, \ldots, p-1 \\
& \mathbf{0}=\frac{\partial \mathcal{L}\left(\boldsymbol{x}^{p *}, \boldsymbol{\sigma}^{*}, \boldsymbol{\lambda}^{*}, \boldsymbol{\rho}^{*}\right)}{\partial \boldsymbol{\sigma}^{i}} \\
& \quad=\int_{\tau_{i-1}}^{\tau_{i}} \quad \frac{\partial H\left(t, \boldsymbol{x}^{p *}, \boldsymbol{\sigma}^{* i}, \boldsymbol{\lambda}^{*}\right)}{\partial \boldsymbol{u}} d t+\left(\boldsymbol{\rho}^{* i}\right)^{\top} \frac{\partial \boldsymbol{\phi}\left(\boldsymbol{\sigma}^{* i}\right)}{\partial \boldsymbol{u}} \\
& \quad i=1, \ldots, p, \\
& \boldsymbol{\phi}\left(\boldsymbol{\sigma}^{* i}\right) \leq \mathbf{0}, \quad i=1, \ldots, p, \\
& \boldsymbol{\rho}^{* i} \geq \mathbf{0}, \quad i=1, \ldots, p, \\
& \rho_{j}^{* i} \phi_{j}\left(\boldsymbol{\sigma}^{* i}\right)=0, \quad j=1, \ldots, q, i=1, \ldots, p .
\end{aligned}
$$

\subsection{Optimal Neighbouring Trajectory}

It should be noted that the optimal control $\sigma^{*}$, the optimal trajectories $\boldsymbol{x}^{p *}$ and $\boldsymbol{\lambda}^{*}$ satisfying the necessary conditions of optimality (17)-(25) are sensitive to disturbances. Now, we consider the neighbouring trajectories of the optimal trajectories under some disturbances. Let $k \in\{1, \ldots, p\}$ be arbitrary but fixed. Define

$$
\begin{aligned}
\overline{\boldsymbol{\sigma}}^{*} & =\left(\boldsymbol{\sigma}^{* k}, \ldots, \boldsymbol{\sigma}^{* p}\right), \\
\overline{\boldsymbol{\rho}}^{*} & =\left(\boldsymbol{\rho}^{* k}, \ldots, \boldsymbol{\rho}^{* p}\right), \\
\boldsymbol{x}^{k *}(t) & =\boldsymbol{x}^{p *}(t), \quad t \in\left[\tau_{k-1}, T\right],
\end{aligned}
$$

and

$$
\lambda^{k *}(t)=\lambda^{*}(t), \quad t \in\left[\tau_{k-1}, T\right] .
$$

We treat $\overline{\boldsymbol{\sigma}}^{*}, \overline{\boldsymbol{\rho}}^{*}, \boldsymbol{x}^{k *}$ and $\boldsymbol{\lambda}^{k *}$ as the functions of the optimal state $\boldsymbol{x}^{k *}\left(\tau_{k-1}\right)$. Thus, they are written as $\overline{\boldsymbol{\sigma}}^{*}\left(\boldsymbol{x}^{k *}\left(\tau_{k-1}\right)\right)$, 
$\overline{\boldsymbol{\rho}}^{*}\left(\boldsymbol{x}^{k *}\left(\tau_{k-1}\right)\right), \boldsymbol{x}^{k *}\left(\cdot \mid \boldsymbol{x}^{k *}\left(\tau_{k-1}\right)\right)$ and $\boldsymbol{\lambda}^{k *}\left(\cdot \mid \boldsymbol{x}^{k *}\left(\tau_{k-1}\right)\right)$. Let $\delta \boldsymbol{x}^{k}\left(\tau_{k-1}\right)$ be a perturbation of the optimal state $\boldsymbol{x}^{k *}\left(\tau_{k-1}\right)$ and $\boldsymbol{h} \in \mathbb{R}^{n}$ be a vector in the direction of $\delta \boldsymbol{x}^{k}\left(\tau_{k-1}\right)$ such that $\delta \boldsymbol{x}^{k}\left(\tau_{k-1}\right)=\epsilon \boldsymbol{h}$ for some $\epsilon \in[0,1]$. For brevity in the remaining part of this paper, we denote $\boldsymbol{x}^{k *}\left(\tau_{k-1}\right)+\epsilon \boldsymbol{h}$ by $\boldsymbol{y}(\epsilon)$. Let $\overline{\boldsymbol{\sigma}}=\overline{\boldsymbol{\sigma}}^{*}(\boldsymbol{y}(\epsilon)), \overline{\boldsymbol{\rho}}=\overline{\boldsymbol{\rho}}^{*}(\boldsymbol{y}(\epsilon))$, $\boldsymbol{x}^{k}(t)=\boldsymbol{x}^{k *}(t \mid \boldsymbol{y}(\epsilon))$ and $\boldsymbol{\lambda}^{k}(t)=\boldsymbol{\lambda}^{k *}(t \mid \boldsymbol{y}(\epsilon))$ be the neighbouring points. We now make the following assumptions.

Assumption 1. The function $\phi$ is twice continuously differentiable, i.e.,

$$
\phi(\cdot) \in C^{2} \text {. }
$$

Assumption 2. If $\phi_{j}\left(\overline{\boldsymbol{\sigma}}^{* l}\left(\boldsymbol{x}^{k *}\left(\tau_{k-1}\right)\right)\right)=0$ for some $l \in$ $\{k, \ldots, p\}$ and $j \in\{1, \ldots, q\}$, then there exists an $\epsilon_{0}>0$ such that $\phi_{j}\left(\overline{\boldsymbol{\sigma}}^{* l}(\boldsymbol{y}(\epsilon))\right)=0$ for all $\epsilon \in\left[0, \epsilon_{0}\right]$ uniformly with respect to $\boldsymbol{h} \in \mathbb{R}^{n}$ and $\|\boldsymbol{h}\| \leq 1$.

Under the above assumptions, it is necessary that conditions (17)-(25) are satisfied for these neighbouring points to remain optimal. Namely,

$$
\begin{aligned}
& \dot{\boldsymbol{x}}^{k}(t)=\sum_{l=k}^{p} \boldsymbol{f}\left(t, \boldsymbol{x}^{k}(t), \overline{\boldsymbol{\sigma}}^{l}\right) \chi_{\left[\tau_{l-1}, \tau_{l}\right)}(t), \\
& \boldsymbol{x}^{k}\left(\tau_{k-1}+\right)=\boldsymbol{y}(\epsilon), \\
& \left(\dot{\boldsymbol{\lambda}}^{k}(t)\right)^{\top}=-\frac{\partial \overline{\mathcal{H}}\left(t, \boldsymbol{x}^{k}, \overline{\boldsymbol{\sigma}}, \boldsymbol{\lambda}^{k}\right)}{\partial \boldsymbol{x}}, \\
& \left(\boldsymbol{\lambda}^{k}(T)\right)^{\top}=\frac{\partial \Phi\left(\boldsymbol{x}^{k}(T)\right)}{\partial \boldsymbol{x}}, \\
& \lambda^{k}\left(\tau_{\iota}-\right)=\boldsymbol{\lambda}^{k}\left(\tau_{\iota}+\right), \quad \iota=k, \ldots, p-1, \\
& \mathbf{0}=\int_{\tau_{l-1}}^{\tau_{l}} \frac{\partial H\left(t, \boldsymbol{x}^{k}, \overline{\boldsymbol{\sigma}}^{l}, \boldsymbol{\lambda}^{k}\right)}{\partial \boldsymbol{u}} d t+\left(\overline{\boldsymbol{\rho}}^{l}\right)^{\top} \frac{\partial \boldsymbol{\phi}\left(\overline{\boldsymbol{\sigma}}^{l}\right)}{\partial \boldsymbol{u}}, \\
& l=k, \ldots, p, \\
& \phi\left(\overline{\boldsymbol{\sigma}}^{l}\right) \leq \mathbf{0}, \quad l=k, \ldots, p, \\
& \overline{\boldsymbol{\rho}}^{l} \geq \mathbf{0}, \quad l=k, \ldots, p, \\
& \bar{\rho}_{j}^{l} \phi_{j}\left(\overline{\boldsymbol{\sigma}}^{l}\right)=0, j=1, \ldots, q, l=k, \ldots, p,
\end{aligned}
$$

where

$$
\overline{\mathcal{H}}\left(t, \boldsymbol{x}^{k}, \overline{\boldsymbol{\sigma}}, \boldsymbol{\lambda}^{k}\right)=\sum_{l=k}^{p} H\left(t, \boldsymbol{x}^{k}, \overline{\boldsymbol{\sigma}}^{l}, \boldsymbol{\lambda}^{k}\right) \chi_{\left[\tau_{l-1}, \tau_{l}\right)}(t) .
$$

By Assumption 1, it is clear that $\phi_{j}\left(\overline{\boldsymbol{\sigma}}^{l *}\right)<0, l=$ $k, \ldots, p$, implies that $\phi_{j}\left(\overline{\boldsymbol{\sigma}}^{l}\right)<0$ if $\epsilon>0$ is sufficiently small. Thus, we have

$$
\bar{\rho}_{j}^{l}=0, \quad j \notin \mathcal{A}^{*}(l)
$$

for $\epsilon>0$ sufficiently small, where

$$
\mathcal{A}^{*}(l)=\mathcal{A}\left(l \mid \sigma^{*}\right)
$$

Also

$$
\begin{aligned}
\left.\frac{d \bar{\rho}_{j}^{l}}{d \epsilon}\right|_{\epsilon=0} & =\frac{\partial \bar{\rho}_{j}^{* l}\left(\boldsymbol{x}^{k *}\left(\tau_{k-1}\right)\right)}{\partial \boldsymbol{y}} \boldsymbol{h} \\
& =0, \quad j \notin \mathcal{A}^{*}(l) .
\end{aligned}
$$

Lemma 1. Let $\boldsymbol{x}^{k}\left(\tau_{k-1}\right)=\boldsymbol{y}(\epsilon), \overline{\boldsymbol{\sigma}}=\overline{\boldsymbol{\sigma}}^{*}(\boldsymbol{y}(\epsilon))$ and $\boldsymbol{\lambda}^{k}(t)=\boldsymbol{\lambda}^{k *}(t \mid \boldsymbol{y}(\epsilon))$ be an optimal neighbouring trajectory for some $\epsilon>0$. Then for each $l=k, \ldots, p$,

$$
\left(\int_{\tau_{l-1}}^{\tau_{l}} \frac{\partial H^{l *}(t)}{\partial \boldsymbol{u}} d t\right) \frac{\partial \overline{\boldsymbol{\sigma}}^{* l}}{\partial \boldsymbol{y}}=\mathbf{0}
$$

where $H^{l *}(t)=H\left(t, \boldsymbol{x}^{k *}, \overline{\boldsymbol{\sigma}}^{* l}, \boldsymbol{\lambda}^{k *}\right)$.

Proof. It follows from (38) that for $l=k, \ldots, p$,

$$
\left(\overline{\boldsymbol{\rho}}^{l}\right)^{\top} \phi\left(\overline{\boldsymbol{\sigma}}^{l}\right)=0 .
$$

Then

$$
\begin{aligned}
0= & \left.\frac{d}{d \epsilon}\left\{\left(\overline{\boldsymbol{\rho}}^{l}\right)^{\top} \boldsymbol{\phi}\left(\overline{\boldsymbol{\sigma}}^{l}\right)\right\}\right|_{\epsilon=0} \\
= & \boldsymbol{h}^{\top}\left(\frac{\partial \overline{\boldsymbol{\rho}}^{* l}}{\partial \boldsymbol{y}}\right)^{\top} \boldsymbol{\phi}\left(\overline{\boldsymbol{\sigma}}^{* l}\right) \\
& +\left(\overline{\boldsymbol{\rho}}^{* l}\right)^{\top} \frac{\partial \boldsymbol{\phi}\left(\overline{\boldsymbol{\sigma}}^{* l}\right)}{\partial \boldsymbol{u}} \frac{\partial \overline{\boldsymbol{\sigma}}^{* l}}{\partial \boldsymbol{y}} .
\end{aligned}
$$

The first term in the above equation is zero since either $\phi_{j}^{l}\left(\overline{\boldsymbol{\sigma}}^{* l}\right)=0$ or (41) holds. Since $\boldsymbol{h} \in \mathbb{R}^{n}$ is arbitrary, we have

$$
\left(\overline{\boldsymbol{\rho}}^{* l}\right)^{\top} \frac{\partial \boldsymbol{\phi}\left(\overline{\boldsymbol{\sigma}}^{* l}\right)}{\partial \boldsymbol{u}} \frac{\partial \overline{\boldsymbol{\sigma}}^{* l}}{\partial \boldsymbol{y}}=\mathbf{0} .
$$

On this basis, the conclusion follows from (22). This completes the proof.

Theorem 1. Let $\boldsymbol{x}^{k}\left(\tau_{k-1}\right)=\boldsymbol{y}(\epsilon), \overline{\boldsymbol{\sigma}}=\overline{\boldsymbol{\sigma}}^{*}(\boldsymbol{y}(\epsilon))$ and $\boldsymbol{\lambda}^{k}(t)=\boldsymbol{\lambda}^{k *}(t \mid \boldsymbol{y}(\epsilon))$ be an optimal neighbouring trajectory for some $\epsilon>0$. Then for each $l=k, \ldots, p$,

$$
\begin{aligned}
& \phi_{j}\left(\overline{\boldsymbol{\sigma}}^{* l}\right)=0, \frac{\partial \phi_{j}\left(\overline{\boldsymbol{\sigma}}^{* l}\right)}{\partial \boldsymbol{u}} \frac{\partial \overline{\boldsymbol{\sigma}}^{* l}}{\partial \boldsymbol{y}}=\mathbf{0}, \text { if } j \in \mathcal{A}^{*}(l), \\
& \bar{\rho}_{j}^{* l}=0, \frac{\partial \bar{\rho}_{j}^{* l}}{\partial \boldsymbol{y}}=\mathbf{0}, \text { if } j \notin \mathcal{A}^{*}(l) .
\end{aligned}
$$

Proof. Suppose that $j \in \mathcal{A}^{*}(l)$. By Assumption 2, there exists $\epsilon_{0}>0$ such that $\phi_{j}\left(\overline{\boldsymbol{\sigma}}^{l}\right)=0$ for all $0 \leq \epsilon \leq \epsilon_{0}$. It follows from the continuity of $\boldsymbol{\phi}$ and $\overline{\boldsymbol{\sigma}}^{* l}(\boldsymbol{y}(\epsilon))$ that

$$
\frac{d}{d \epsilon}\left\{\left.\phi_{j}\left(\overline{\boldsymbol{\sigma}}^{* l}(\boldsymbol{y}(\epsilon))\right\}\right|_{\epsilon=0}=0 .\right.
$$

Since $h$ is arbitrary, (43) follows.

Suppose that $j \notin \mathcal{A}^{*}(l)$, then $\bar{\rho}_{j}^{* l}(\boldsymbol{y}(\epsilon))=0$. Since $\overline{\boldsymbol{\rho}}^{* l}$ is continuous at $\boldsymbol{y},(44)$ follows by a similar arguments.

Theorem 2. Let $\boldsymbol{x}^{k}\left(\tau_{k-1}\right)=\boldsymbol{y}(\epsilon), \overline{\boldsymbol{\sigma}}=\overline{\boldsymbol{\sigma}}^{*}(\boldsymbol{y}(\epsilon))$ and $\boldsymbol{\lambda}^{k}(t)=\boldsymbol{\lambda}^{k *}(t \mid \boldsymbol{y}(\epsilon))$ be an optimal neighbouring trajectory for some $\epsilon>0$. Then for each $l=k, \ldots, p$,

$$
\begin{aligned}
& \int_{\tau_{l-1}}^{\tau_{l}}\left\{\frac{\partial^{2} H^{l *}(t)}{\partial \boldsymbol{u} \partial \boldsymbol{x}} \frac{\partial \boldsymbol{x}^{k *}(t)}{\partial \boldsymbol{y}}+\left(\frac{\partial \boldsymbol{f}^{l *}(t)}{\partial \boldsymbol{u}}\right)^{\top} \frac{\partial \boldsymbol{\lambda}^{k *}(t)}{\partial \boldsymbol{y}}\right\} d t \\
& +\left(\int_{\tau_{l-1}}^{\tau_{l}} \frac{\partial^{2} H^{l *}(t)}{\partial \boldsymbol{u}^{2}} d t+\sum_{j \in \mathcal{A}^{*}(l)} \bar{\rho}_{j}^{* l} \frac{\partial^{2} \phi_{j}\left(\overline{\boldsymbol{\sigma}}^{* l}\right)}{\partial \boldsymbol{u}^{2}}\right) \frac{\partial \overline{\boldsymbol{\sigma}}^{* l}}{\partial \boldsymbol{y}} \\
& +\sum_{j \in \mathcal{A}^{*}(l)}\left(\frac{\partial \phi_{j}\left(\overline{\boldsymbol{\sigma}}^{* l}\right)}{\partial \boldsymbol{u}}\right)^{\top} \frac{\partial \bar{\rho}_{j}^{* l}}{\partial \boldsymbol{y}}=\mathbf{0},
\end{aligned}
$$


where $\boldsymbol{f}^{l *}(t)=\boldsymbol{f}\left(t, \boldsymbol{x}^{k *}(t), \overline{\boldsymbol{\sigma}}^{* l}\right)$, and

$$
\frac{\partial^{2} H^{l *}(t)}{\partial \boldsymbol{u} \partial \boldsymbol{x}}=\left[\begin{array}{cccc}
\frac{\partial^{2} H^{l *}(t)}{\partial u_{1} \partial x_{1}} & \frac{\partial^{2} H^{l *}(t)}{\partial u_{1} \partial x_{2}} & \ldots & \frac{\partial^{2} H^{l *}(t)}{\partial u_{1} \partial x_{n}} \\
\frac{\partial^{2} H^{l *}}{\partial u_{2} \partial x_{1}} & \frac{\partial^{2} H^{l *}(t)}{\partial u_{2} \partial x_{2}} & \ldots & \frac{\partial^{2} H^{l *}(t)}{\partial u_{2} \partial x_{n}} \\
\vdots & \vdots & \ddots & \vdots \\
\frac{\partial^{2} H^{l *}(t)}{\partial u_{m} \partial x_{1}} & \frac{\partial^{2} H^{l *}(t)}{\partial u_{m} \partial x_{2}} & \cdots & \frac{\partial^{2} H^{l *}(t)}{\partial u_{m} \partial x_{n}}
\end{array}\right] .
$$

Proof. Since $\left(\boldsymbol{x}^{k}, \overline{\boldsymbol{\sigma}}, \boldsymbol{\lambda}^{k}\right)$ is optimal, it follows that for $l=$ $k, \ldots, p$,

$$
\int_{\tau_{l-1}}^{\tau_{l}} \frac{\partial H^{l}(t)}{\partial \boldsymbol{u}} d t+\sum_{j=1}^{q} \bar{\rho}_{j}^{l} \frac{\partial \phi_{j}\left(\overline{\boldsymbol{\sigma}}^{l}\right)}{\partial \boldsymbol{u}}=\mathbf{0}
$$

where $H^{l}(t)=H\left(t, \boldsymbol{x}^{k}, \overline{\boldsymbol{\sigma}}^{l}, \boldsymbol{\lambda}^{k}\right)$. Thus,

$$
\begin{aligned}
\mathbf{0}= & \left.\int_{\tau_{l-1}}^{\tau_{l}} \frac{d}{d \epsilon}\left\{\frac{\partial H^{l}(t)}{\partial \boldsymbol{u}}\right\}^{\top}\right|_{\epsilon=0} d t+\sum_{j=1}^{q}\left(\frac{d \bar{\rho}_{j}^{l}}{d \epsilon} \frac{\partial \phi_{j}\left(\overline{\boldsymbol{\sigma}}^{l}\right)}{\partial \boldsymbol{u}}\right. \\
& \left.+\bar{\rho}_{j}^{l} \frac{d}{d \epsilon}\left\{\frac{\partial \phi_{j}\left(\overline{\boldsymbol{\sigma}}^{l}\right)}{\partial \boldsymbol{u}}\right\}\right)\left.^{\top}\right|_{\epsilon=0} \\
= & \int_{\tau_{l-1}}^{\tau_{l}}\left(\frac{\partial^{2} H^{l *}(t)}{\partial \boldsymbol{u} \partial \boldsymbol{x}} \frac{\partial \boldsymbol{x}^{k *}(t)}{\partial \boldsymbol{y}}+\left(\frac{\partial \boldsymbol{f}^{l *}(t)}{\partial \boldsymbol{u}}\right)^{\top}\right. \\
& \left.\times \frac{\partial \boldsymbol{\lambda}^{k *}(t)}{\partial \boldsymbol{y}}\right) \boldsymbol{h} d t+\left(\int_{\tau_{l-1}}^{\tau_{l}} \frac{\partial^{2} H^{l *}(t)}{\partial \boldsymbol{u}^{2}} d t\right) \frac{\partial \overline{\boldsymbol{\sigma}}^{* l}}{\partial \boldsymbol{y}} \boldsymbol{h} \\
& +\sum_{j=1}^{q}\left(\left(\frac{\partial \phi_{j}\left(\overline{\boldsymbol{\sigma}}^{* l}\right)}{\partial \boldsymbol{u}}\right)^{\top} \frac{\partial \bar{\rho}_{j}^{* l}}{\partial \boldsymbol{y}}\right. \\
& \left.+\bar{\rho}_{j}^{* l} \frac{\partial^{2} \phi_{j}\left(\overline{\boldsymbol{\sigma}}^{* l}\right)}{\partial \boldsymbol{u}^{2}} \frac{\partial \overline{\boldsymbol{\sigma}}^{* l}}{\partial \boldsymbol{y}}\right) \boldsymbol{h} .
\end{aligned}
$$

Since $\boldsymbol{h}$ is arbitrary, (45) follows from (16) and (44). This completes the proof.

Theorem 3. Let $\boldsymbol{x}^{k}\left(\tau_{k-1}\right)=\boldsymbol{y}(\epsilon), \overline{\boldsymbol{\sigma}}=\overline{\boldsymbol{\sigma}}^{*}(\boldsymbol{y}(\epsilon))$ and $\lambda^{k}(t)=\lambda^{k *}(t \mid \boldsymbol{y}(\epsilon))$ be an optimal neighbouring trajectory for some $\epsilon>0$. Then

$$
\begin{aligned}
\frac{\partial \dot{\boldsymbol{x}}^{k *}(t)}{\partial \boldsymbol{y}}= & \frac{\partial \boldsymbol{f}^{l *}(t)}{\partial \boldsymbol{x}} \frac{\partial \boldsymbol{x}^{k *}(t)}{\partial \boldsymbol{y}}+\frac{\partial \boldsymbol{f}^{l *}(t)}{\partial \boldsymbol{u}} \frac{\partial \overline{\boldsymbol{\sigma}}^{* l}}{\partial \boldsymbol{y}} \\
\frac{\partial \dot{\boldsymbol{\lambda}}^{k *}(t)}{\partial \boldsymbol{y}}= & -\frac{\partial^{2} H^{l *}(t)}{\partial \boldsymbol{x}^{2}} \frac{\partial \boldsymbol{x}^{k *}(t)}{\partial \boldsymbol{y}}-\frac{\partial^{2} H^{l *}(t)}{\partial \boldsymbol{x} \partial \boldsymbol{u}} \frac{\partial \overline{\boldsymbol{\sigma}}^{* l}}{\partial \boldsymbol{y}} \\
& -\left(\frac{\partial \boldsymbol{f}^{l *}(t)}{\partial \boldsymbol{x}}\right)^{\top} \frac{\partial \boldsymbol{\lambda}^{k *}(t)}{\partial \boldsymbol{y}} \\
& t \in\left[\tau_{l-1}, \tau_{l}\right), \quad l=k, \ldots, p
\end{aligned}
$$

with the following conditions:

$$
\begin{aligned}
\frac{\partial \boldsymbol{x}^{k *}\left(\tau_{k-1}\right)}{\partial \boldsymbol{y}} & =\mathbf{1}, \\
\frac{\partial \boldsymbol{\lambda}^{k *}(T)}{\partial \boldsymbol{y}} & =\frac{\partial^{2} \Phi\left(\boldsymbol{x}^{k *}(T)\right)}{\partial \boldsymbol{x}^{2}} \frac{\partial \boldsymbol{x}^{k *}(T)}{\partial \boldsymbol{y}}, \\
\frac{\partial \boldsymbol{x}^{k *}\left(\tau_{\iota}+\right)}{\partial \boldsymbol{y}} & =\frac{\partial \boldsymbol{x}^{k *}\left(\tau_{\iota}-\right)}{\partial \boldsymbol{y}}, \\
\frac{\partial \boldsymbol{\lambda}^{k *}\left(\tau_{\iota}-\right)}{\partial \boldsymbol{y}} & =\frac{\partial \boldsymbol{\lambda}^{k *}\left(\tau_{\iota}+\right)}{\partial \boldsymbol{y}}, \quad \iota=k, \ldots, p-1,
\end{aligned}
$$

where 1 is an unit matrix of order $n$.
To solve system (46)-(47), we use a modification of the backward sweep method [8]. First we must express $\frac{\partial \boldsymbol{\lambda}^{k *}(t)}{\partial \boldsymbol{y}}$ in terms of $\frac{\partial \boldsymbol{x}^{k *}(t)}{\partial \boldsymbol{y}}$ and $\frac{\partial \overline{\boldsymbol{\sigma}}^{*}}{\partial \boldsymbol{y}}$ as given in the following theorem:

Theorem 4. Let $\boldsymbol{x}^{k}\left(\tau_{k-1}\right)=\boldsymbol{y}(\epsilon), \overline{\boldsymbol{\sigma}}=\overline{\boldsymbol{\sigma}}^{*}(\boldsymbol{y}(\epsilon))$ and $\boldsymbol{\lambda}^{k}(t)=\boldsymbol{\lambda}^{k *}(t \mid \boldsymbol{y}(\epsilon))$ be an optimal neighbouring trajectory for some $\epsilon>0$. Then

$$
\begin{aligned}
\frac{\partial \boldsymbol{\lambda}^{k *}(t)}{\partial \boldsymbol{y}}=A(t) \frac{\partial \boldsymbol{x}^{k *}(t)}{\partial \boldsymbol{y}}+B(t) \frac{\partial \overline{\boldsymbol{\sigma}}^{* l}}{\partial \boldsymbol{y}}, \\
t \in\left[\tau_{l-1}, \tau_{l}\right), \quad l=k, \ldots, p,
\end{aligned}
$$

where $A(t)$ and $B(t)$ are, respectively, $n \times n$ and $n \times m$ matrices that satisfy the following differential relations:

$$
\begin{gathered}
\dot{A}(t)=-\left(\frac{\partial \boldsymbol{f}^{l *}(t)}{\partial \boldsymbol{x}}\right)^{\top} A(t)-A(t) \frac{\partial \boldsymbol{f}^{l *}(t)}{\partial \boldsymbol{x}}-\frac{\partial^{2} H^{l *}(t)}{\partial \boldsymbol{x}^{2}}, \\
\dot{B}(t)=-\left(\frac{\partial \boldsymbol{f}^{l *}(t)}{\partial \boldsymbol{x}}\right)^{\top} B(t)-A(t) \frac{\partial \boldsymbol{f}^{l *}(t)}{\partial \boldsymbol{u}}-\frac{\partial^{2} H^{l *}(t)}{\partial \boldsymbol{x} \partial \boldsymbol{u}}, \\
t \in\left[\tau_{l-1}, \tau_{l}\right), \quad l=k, \ldots, p,
\end{gathered}
$$

with the intermediate and terminal conditions:

$$
\begin{aligned}
A(T) & =\frac{\partial^{2} \Phi\left(\boldsymbol{x}^{k *}(T)\right)}{\partial \boldsymbol{x}^{2}}, \\
B(T) & =\mathbf{0}, \\
A\left(\tau_{\iota}-\right) & =A\left(\tau_{\iota}+\right), \quad \iota=k, \ldots, p-1, \\
B\left(\tau_{\iota}-\right) & =B\left(\tau_{\iota}+\right), \quad \iota=k, \ldots, p-1 .
\end{aligned}
$$

Proof. To find (53) and (54), we differentiate (52) with respect to time

$$
\begin{gathered}
\frac{\partial \dot{\boldsymbol{\lambda}}^{k *}(t)}{\partial \boldsymbol{y}}=\dot{A}(t) \frac{\partial \boldsymbol{x}^{k *}(t)}{\partial \boldsymbol{y}}+A(t) \frac{\partial \dot{\boldsymbol{x}}^{k *}(t)}{\partial \boldsymbol{y}}+\dot{B}(t) \frac{\partial \overline{\boldsymbol{\sigma}}^{* l}}{\partial \boldsymbol{y}}, \\
t \in\left[\tau_{l-1}, \tau_{l}\right), \quad l=k, \ldots, p .
\end{gathered}
$$

Substituting (46) and (47) into (59) yields

$$
\begin{aligned}
& -\frac{\partial^{2} H^{l *}(t)}{\partial \boldsymbol{x}^{2}} \frac{\partial \boldsymbol{x}^{k *}(t)}{\partial \boldsymbol{y}}-\frac{\partial^{2} H^{l *}(t)}{\partial \boldsymbol{x} \partial \boldsymbol{u}} \frac{\partial \overline{\boldsymbol{\sigma}}^{* l}}{\partial \boldsymbol{y}} \\
& -\left(\frac{\partial \boldsymbol{f}^{l *}(t)}{\partial \boldsymbol{x}}\right)^{\top} \frac{\partial \boldsymbol{\lambda}^{k *}(t)}{\partial \boldsymbol{y}} \\
& =\dot{A}(t) \frac{\partial \boldsymbol{x}^{k *}(t)}{\partial \boldsymbol{y}}+A(t) \frac{\partial \boldsymbol{f}^{l *}(t)}{\partial \boldsymbol{x}} \frac{\partial \boldsymbol{x}^{k *}(t)}{\partial \boldsymbol{y}} \\
& +\dot{B}(t) \frac{\partial \overline{\boldsymbol{\sigma}}^{* l}}{\partial \boldsymbol{y}} .
\end{aligned}
$$

Then, substituting (52) into the equation gives

$$
\begin{gathered}
-\frac{\partial^{2} H^{l *}(t)}{\partial \boldsymbol{x}^{2}} \frac{\partial \boldsymbol{x}^{k *}(t)}{\partial \boldsymbol{y}}-\frac{\partial^{2} H^{l *}(t)}{\partial \boldsymbol{x} \partial \boldsymbol{u}} \frac{\partial \overline{\boldsymbol{\sigma}}^{* l}}{\partial \boldsymbol{y}} \\
-\left(\frac{\partial \boldsymbol{f}^{l *}(t)}{\partial \boldsymbol{x}}\right)^{\top} A(t) \frac{\partial \boldsymbol{x}^{k *}(t)}{\partial \boldsymbol{y}}-\left(\frac{\partial \boldsymbol{f}^{l *}(t)}{\partial \boldsymbol{x}}\right)^{\top} B(t) \frac{\partial \overline{\boldsymbol{\sigma}}^{* l}}{\partial \boldsymbol{y}} \\
=\dot{A}(t) \frac{\partial \boldsymbol{x}^{k *}(t)}{\partial \boldsymbol{y}}+A(t) \frac{\partial \boldsymbol{f}^{l *}(t)}{\partial \boldsymbol{x}} \frac{\partial \boldsymbol{x}^{k *}(t)}{\partial \boldsymbol{y}} \\
+A(t) \frac{\partial \boldsymbol{f}^{l *}(t)}{\partial \boldsymbol{u}} \frac{\partial \overline{\boldsymbol{\sigma}}^{* l}}{\partial \boldsymbol{y}}+\dot{B}(t) \frac{\partial \overline{\boldsymbol{\sigma}}^{* l}}{\partial \boldsymbol{y}} .
\end{gathered}
$$


Since $\frac{\partial \boldsymbol{x}^{k *}(t)}{\partial \boldsymbol{y}}$ and $\frac{\partial \overline{\boldsymbol{\sigma}}^{* l}}{\partial \boldsymbol{y}}$ are chosen arbitrarily, we obtained (53) and (54). The intermediate and terminal conditions (55)-(58) can be verified according to (49)-(51).

\section{Neigbouring Feedback Control Law}

From Theorem 4, we can obtain $\frac{\partial \boldsymbol{\lambda}^{k *}\left(\tau_{k-1}\right)}{\partial \boldsymbol{y}}$. Using this result, we develop a procedure that can be used to determine $\frac{\partial \overline{\boldsymbol{\sigma}}^{*}}{\partial \boldsymbol{y}}$ as follows.

Let us rewrite (46) and (47) as

$$
\begin{aligned}
& \dot{\boldsymbol{z}}(t)=C^{l}(t) \boldsymbol{z}(t)+D^{l}(t) \boldsymbol{\theta}^{l}, \quad t \in\left(\tau_{l-1}, \tau_{l}\right], \\
& l=k, \ldots, p, \\
& \boldsymbol{z}\left(\tau_{k-1}\right)=E^{k}+F^{k} \boldsymbol{\theta}^{k},
\end{aligned}
$$

where

$$
\begin{aligned}
& \boldsymbol{z}(t)=\left[\left(\frac{\partial \boldsymbol{x}^{k *}(t)}{\partial \boldsymbol{y}}\right)^{\top},\left(\frac{\partial \boldsymbol{\lambda}^{k *}(t)}{\partial \boldsymbol{y}}\right)^{\top}\right]^{\top}, \\
& \boldsymbol{\theta}=\left(\boldsymbol{\theta}^{k}, \ldots, \boldsymbol{\theta}^{p}\right)=\left[\frac{\partial \overline{\boldsymbol{\sigma}}^{* k}}{\partial \boldsymbol{y}}, \ldots, \frac{\partial \overline{\boldsymbol{\sigma}}^{* p}}{\partial \boldsymbol{y}}\right] \\
& C^{l}(t)=\left[\begin{array}{cc}
\frac{\partial \boldsymbol{f}^{l *}(t)}{\partial \boldsymbol{x}} & \mathbf{0} \\
-\frac{\partial^{2} H^{l *}(t)}{\partial \boldsymbol{x}^{2}} & -\left(\frac{\partial \boldsymbol{f}^{l *}(t)}{\partial \boldsymbol{x}}\right)^{\top}
\end{array}\right], \\
& D^{l}(t)=\left[\begin{array}{c}
\frac{\partial \boldsymbol{f}^{l *}(t)}{\partial \boldsymbol{u}^{l *}} \\
-\frac{\partial^{2} H^{l *}(t)}{\partial \boldsymbol{x} \partial \boldsymbol{u}}
\end{array}\right], \quad E^{k}=\left[\begin{array}{c}
\mathbf{1} \\
A\left(\tau_{k-1}\right)
\end{array}\right], \\
& F^{k}=\left[\begin{array}{c}
\mathbf{0} \\
B\left(\tau_{k-1}\right)
\end{array}\right] .
\end{aligned}
$$

Let $\hat{\boldsymbol{\rho}}^{* l}$ and $\hat{\boldsymbol{\phi}}^{* l}$ be vectors formed from the components of $\overline{\boldsymbol{\rho}}^{* l}$ and $\phi\left(\overline{\boldsymbol{\sigma}}^{* l}\right)$ corresponding to the active constraints. For each $l=k, \ldots, p$, equations (43) and (45) can now be combined to give the following matrix equation:

$$
\left[\begin{array}{cc}
\tilde{A}^{l *} & \tilde{B}^{l *} \\
\left(\tilde{B}^{l *}\right)^{\top} & \mathbf{0}
\end{array}\right]\left[\begin{array}{c}
\boldsymbol{\theta}^{l} \\
\frac{\partial \hat{\boldsymbol{\rho}}^{* l}}{\partial \boldsymbol{y}}
\end{array}\right]=\left[\begin{array}{c}
\tilde{E}^{l *} \\
\mathbf{0}
\end{array}\right]
$$

where

$$
\begin{aligned}
\tilde{A}^{l *} & =\int_{\tau_{l-1}}^{\tau_{l}} \frac{\partial^{2} H^{l *}(t)}{\partial \boldsymbol{u}^{2}} d t+\sum_{j \in \mathcal{A}^{*}(l)} \bar{\rho}_{j}^{* l} \frac{\partial^{2} \phi_{j}\left(\overline{\boldsymbol{\sigma}}^{* l}\right)}{\partial \boldsymbol{u}^{2}}, \\
\tilde{B}^{l *} & =\left(\frac{\partial \hat{\boldsymbol{\phi}}^{* l}}{\partial \boldsymbol{u}}\right)^{\top}, \\
\tilde{E}^{l *} & =-\int_{\tau_{l-1}}^{\tau_{l}}\left[\frac{\partial^{2} H^{l *}(t)}{\partial \boldsymbol{u} \partial \boldsymbol{x}},\left(\frac{\partial \boldsymbol{f}^{l *}(t)}{\partial \boldsymbol{u}}\right)^{\top}\right] \boldsymbol{z}(t) d t .
\end{aligned}
$$

This is a system of $\left(m+\left|\mathcal{A}^{*}(l)\right|\right) n$ linear equations in the $\left(m+\left|\mathcal{A}^{*}(l)\right|\right) n$ unknowns $\boldsymbol{\theta}^{l}$ and $\partial \hat{\boldsymbol{\rho}}^{* l} / \partial \boldsymbol{y}$. If $\tilde{A}^{l *}$ and $\left(\tilde{B}^{l *}\right)^{\top}\left(\tilde{A}^{l *}\right)^{-1} \tilde{B}^{l *}$ are both invertible then $(63)$ can be solved explicitly to give

$$
\begin{aligned}
& \left(\tilde{A}^{l *}\right)^{-1} \tilde{B}^{l *}\left[\left(\tilde{B}^{l *}\right)^{\top}\left(\tilde{A}^{l *}\right)^{-1} \tilde{B}^{l *}\right]^{-1}\left(\tilde{B}^{l *}\right)^{\top}\left(\tilde{A}^{l *}\right)^{-1} \tilde{E}^{l *} \\
& \quad-\left(\tilde{A}^{l *}\right)^{-1} \tilde{E}^{l *}+\boldsymbol{\theta}^{l}=\mathbf{0} .
\end{aligned}
$$

Equation (64) can be regarded as constraints to solve system (61)-(62). To determine $\boldsymbol{\theta}^{l}$ satisfying (64), the gradients of the solution $\boldsymbol{z}(t)$ with respect to $\boldsymbol{\theta}^{l}$ are needed, which are given in the following theorem.

Theorem 5. For each $\ell \in\{k, \ldots, p\}$,

$$
\frac{\partial \boldsymbol{z}(t)}{\partial \boldsymbol{\theta}^{\ell}}=\Gamma(t), \quad t \in\left[\tau_{l-1}, \tau_{l}\right], \quad l=k, \ldots, p,
$$

where $\Gamma(\cdot)$ is the solution of the following auxiliary system:

$$
\dot{\Gamma}(s)= \begin{cases}C^{\ell}(s) \Gamma(s)+D^{\ell}(s), & s \in\left(\tau_{\ell-1}, \tau_{\ell}\right], \\ C^{l}(s) \Gamma(s), & s \in\left(\tau_{l-1}, \tau_{l}\right], \quad l \neq \ell,\end{cases}
$$

with the initial condition

$$
\Gamma\left(\tau_{k-1}\right)= \begin{cases}F^{k}, & \ell=k \\ \mathbf{0}, & \text { otherwise. }\end{cases}
$$

Now, if an initial guess of $\boldsymbol{\theta}^{l}$ is $\boldsymbol{\theta}_{e}^{l}$ which does not satisfy the constraint (64), then

$$
\begin{aligned}
\Omega_{e}= & \left(\tilde{A}^{l *}\right)^{-1} \tilde{B}^{l *}\left[\left(\tilde{B}^{l *}\right)^{\top}\left(\tilde{A}^{l *}\right)^{-1} \tilde{B}^{l *}\right]^{-1}\left(\tilde{B}^{l *}\right)^{\top} \\
& \times\left(\tilde{A}^{l *}\right)^{-1} \tilde{E}^{l *}-\left(\tilde{A}^{l *}\right)^{-1} \tilde{E}^{l *}+\boldsymbol{\theta}_{e}^{l} \neq \mathbf{0} .
\end{aligned}
$$

To satisfy this, we change the initial guess $\boldsymbol{\theta}_{e}^{l}$ to $\boldsymbol{\theta}_{e}^{l}+\delta \boldsymbol{\theta}^{l}$. Since the constraint (64) is now satisfied exactly, we have

$$
\begin{aligned}
& \left(\tilde{A}^{l *}\right)^{-1} \tilde{B}^{l *}\left[\left(\tilde{B}^{l *}\right)^{\top}\left(\tilde{A}^{l *}\right)^{-1} \tilde{B}^{l *}\right]^{-1}\left(\tilde{B}^{l *}\right)^{\top}\left(\tilde{A}^{l *}\right)^{-1} \tilde{\tilde{E}}^{l *} \\
& -\left(\tilde{A}^{l *}\right)^{-1} \tilde{\tilde{E}}^{l *}+\boldsymbol{\theta}_{e}^{l}+\delta \boldsymbol{\theta}^{l}=\mathbf{0}
\end{aligned}
$$

where

$$
\begin{aligned}
\tilde{\tilde{E}}^{l *}=- & \int_{\tau_{l-1}}^{\tau_{l}}\left\{\left[\frac{\partial^{2} H^{l *}(t)}{\partial \boldsymbol{u} \partial \boldsymbol{x}},\left(\frac{\partial \boldsymbol{f}^{l *}(t)}{\partial \boldsymbol{u}}\right)^{\top}\right]\right. \\
& \left.\times\left(\boldsymbol{z}(t)+\frac{\partial \boldsymbol{z}(t)}{\partial \boldsymbol{\theta}^{l}} \delta \boldsymbol{\theta}^{l}\right)\right\} d t .
\end{aligned}
$$

The difference between (66) and (67) is

$$
\Omega_{e}=K \delta \boldsymbol{\theta}^{l},
$$

where

$$
\begin{aligned}
K= & \left(\tilde{A}^{l *}\right)^{-1} \tilde{B}^{l *}\left[\left(\tilde{B}^{l *}\right)^{\top}\left(\tilde{A}^{l *}\right)^{-1} \tilde{B}^{l *}\right]^{-1}\left(\tilde{B}^{l *}\right)^{\top}\left(\tilde{A}^{l *}\right)^{-1} \\
& \times\left\{\int_{\tau_{l-1}}^{\tau_{l}}\left[\frac{\partial^{2} H^{l *}(t)}{\partial \boldsymbol{u} \partial \boldsymbol{x}},\left(\frac{\partial \boldsymbol{f}^{l *}(t)}{\partial \boldsymbol{u}}\right)^{\top}\right] \frac{\partial \boldsymbol{z}(t)}{\partial \boldsymbol{\theta}^{l}} d t\right\} \\
& -\left(\tilde{A}^{l *}\right)^{-1}\left\{\int_{\tau_{l-1}}^{\tau_{l}}\left[\frac{\partial^{2} H^{l *}(t)}{\partial \boldsymbol{u} \partial \boldsymbol{x}},\left(\frac{\partial \boldsymbol{f}^{l *}(t)}{\partial \boldsymbol{u}}\right)^{\top}\right] \frac{\partial \boldsymbol{z}(t)}{\partial \boldsymbol{\theta}^{l}} d t\right\} \\
& -\mathbf{1}
\end{aligned}
$$

Therefore

$$
\delta \boldsymbol{\theta}^{l}=K^{-1} \Omega_{e} .
$$

Now, we give the main result in the following theorem, which gives neighbouring control law in a state feedback form.

Theorem 6. Let $\delta \boldsymbol{x}^{k}\left(\tau_{k-1}\right)=\epsilon \boldsymbol{h}$ be a perturbation of the optimal reference state $\boldsymbol{x}^{k *}\left(\tau_{k-1}\right)$ for some $\epsilon \in[0,1]$ and $\boldsymbol{y}(\epsilon)=\boldsymbol{x}^{k *}\left(\tau_{k-1}\right)+\epsilon \boldsymbol{h}$. Then

$$
\overline{\boldsymbol{\sigma}}^{l}(\boldsymbol{y}(\epsilon))=\overline{\boldsymbol{\sigma}}^{* l}+\frac{\partial \overline{\boldsymbol{\sigma}}^{* l}}{\partial \boldsymbol{y}} \epsilon \boldsymbol{h}, l=k, \ldots, p .
$$




\section{Conclusions}

In this paper, we developed an approach computing the neighbouring extremals for optimal control problems with control constraints. The optimal control problems was first solved by control parameterization method to obtain the optimal open-loop control. Then, a neighbouring feedback control law was constructed using the neighbouring extremal method.

Further work still needs to be done to test the proposed computational approach using numerical examples.

\section{References}

[1] L. Hasdorff, Gradient Optimization and Nonlinear Control, New York: John Wiley and Sons, 1976.

[2] H.R. Sirisena, F.S. Chou, State parameterization approach to the solution of optimal control problems, Optimal Control Applications and Methods, 2:289-298, 1981.

[3] O. von Stryk, R. Bulirsch, Direct and indirect methods for trajectory optimization, Annals of Operations Research, 37:357373, 1992.

[4] K.L. Teo, C.J. Goh, K.H. Wong, A Unified Computational Approach to Optimal Control Problems, Essex: Long Scientific Technical, 1991.

[5] Q. Lin, R. Loxton, K.L. Teo, The control parameterization method for nonlinear optimal control: A survey, Journal of Industrial Management and Optimization, 10:275-309, 2014.

[6] J. Nocedal, S.J. Wright, Numerical Optimization, New York: Springer-Verlag, 1999.

[7] R. Bellman, Dynamic Programming, New Jersey: Princeton University Press, 1972.

[8] A.E. Bryson, Y.C. Ho, Applied Optimal Control, Washington: Hemisphere, 1975.

[9] A.E. Breakwell, J.L. Speyer, A.E. Bryson, Optimization and control of nonlinear systems using the second variation, SIAM Journal of Control and Optimization, 1:193-217, 1963.

[10] A.Y. Lee, A.E. Bryson, Neighbouring extremals of dynamic optimization problems with parameter variations, Optimal Control Applications and Methods, 10:39-52, 1989.

[11] M.E. Fisher, W.J. Grantham, K.L. Teo, Neighbouring extremals for nonlinear systems with control constraints, Dynamics and Control, 5:225-240, 1995.

[12] B. Kugelmann, H.J. Pesch, A new general guidance method in constrained optimal control Part I: Numerical method, Journal of Optimization Theory \& Applications, 67:421-435, 1990.

[13] K. Malanowski, H. Maurer, Sensitivity analysis for parametric control problems with control-state constraints, Computational Optimization and Applications, 5:253-283, 1996.

[14] K. Malanowski, H. Maurer, Sensitivity analysis for state constrained optimal control problems, Discrete and Continuous Dynamic Systems, 4:241-272, 1998.

[15] C. Büskens, H. Maurer, SQP-methods for solving optimal control problems with control and state constraints: Adjoint variables, sensitivity analysis and real-time control, Journal of Computational and Applied Mathematics, 120:85-108, 2000.

[16] C. Jiang, K.L. Teo, G. Duan, A suboptimal feedback control for nonlinear time-varying systems with continous inequality constraints, Automatica, 48:660-665, 2012. 